\title{
Studies of the neutron spin structure at Jefferson Lab
}

\author{
W. Korsch ${ }^{\mathrm{a}}$ \\ Physics and Astronomy, University of Kentucky, 177 Chem.-Phys. Bldg., Lexington, KY 40506, USA
}

Received: 1 November 2002 /

Published online: 15 July 2003 - C Società Italiana di Fisica / Springer-Verlag 2003

\begin{abstract}
The polarized ${ }^{3} \mathrm{He}$ program of Hall A at Jefferson Lab will be described. Results on the generalized Gerasimov-Drell-Hearn integral for the neutron in a $Q^{2}$ range between $0.02 \mathrm{GeV}^{2} / c^{2}<Q^{2}<$ $0.9 \mathrm{GeV}^{2} / c^{2}$ will be presented. Preliminary results of the virtual photon asymmetry $A_{1}^{n}\left(x, Q^{2}\right)$ and the spin structure function $g_{2}^{n}\left(x, Q^{2}\right)$ at large values of Bjorken $x$ and low $Q^{2}$, respectively, will be discussed.

PACS. 11.55.Hx Sum rules - 13.40.Gp Electromagnetic form factors - 13.88.+e Polarization in interactions and scattering - 29.25.Pj Polarized and other targets
\end{abstract}

\section{Introduction}

For more than a decade most major accelerator laboratories in the world have carried out sophisticated experiments to study the fundamental spin structure of the nucleons. Contrary to the early expectation that the spin is completely carried by the valence quarks of the nucleon, contributions from valence and sea quarks, as well as the gluons and possible orbital excitations are equally important. A vast amount of experimental and theoretical effort has been devoted to disentangle these different contributions [1]. It turns out that often sum rules, i.e. integrals over certain physical quantities, allow for a very robust comparison between experiment and theory. One of the most prominent examples is the Bjorken (Bj) sum rule which relates the axial charge, $(\Delta u+\Delta \bar{u})-(\Delta d+\Delta \bar{d})=$ $g_{A} / g_{V}$, to the difference of the first moments of the proton and neutron spin structure function $g_{1}^{p}$ and $g_{1}^{n}$, respectively [2]. This sum rule is predicted to be $0.181 \pm 0.005$ (up to $\alpha_{s}^{3}$ corrections) at a $Q^{2}$ of $5 \mathrm{GeV}^{2} / c^{2}$. It has been experimentally verified to within $5 \%$. This result is one of the most direct tests of perturbative QCD. Another sum rule, the Gerasimov-Drell-Hearn (GDH) sum rule, has also gained recent popularity. This sum rule relates the difference of the spin-dependent photon-nucleon cross-sections to the anomalous magnet moment of the nucleon. It was derived in 1966 by Gerasimov [3] and, independently, by Drell and Hearn [3], based on very fundamental principles. Experimentally, such integrals are very difficult to evaluate, especially when they are $Q^{2}$-dependent. Evolutions and extrapolations are often necessary. With the advent of highly polarized few-nucleon targets and highly polarized c.w. beams, e.g. at Jefferson Lab, MAMI or ELSA,

\footnotetext{
a e-mail: korsch@pa.uky.edu
}

at least the low-energy contribution to some integrals can finally be addressed with high precision.

In addition to sum rules precision measurements of the spin structure functions, $g_{1}\left(x, Q^{2}\right)$ and $g_{2}\left(x, Q^{2}\right)$, and, more recently, generalized parton distributions and transversity have attracted considerable interest.

The focus of this paper is on the $Q^{2}$ evolution of the GDH sum for low and intermediate values of $Q^{2}$ (sect. 2). Precision measurements of inclusive asymmetries and the extraction of spin structure functions in previously unexplored kinematical regions such as large values of Bjorken $x$ or low values of $Q^{2}$ will be discussed in sects. 3 and 4 .

\section{The GDH integral for the neutron}

All experiments described here were performed using inclusive polarized electron-nucleon scattering. In such experiments two independent structure functions, $g_{1}\left(x, Q^{2}\right)$ and $g_{2}\left(x, Q^{2}\right)$, can be isolated by measuring the following scattering asymmetries:

$$
\begin{gathered}
A_{\|}=\frac{\sigma^{\rightleftarrows}-\sigma^{\rightrightarrows}}{\sigma \rightleftarrows+\sigma \rightrightarrows}=D \cdot\left\{\left(E+E^{\prime} \cos (\theta)\right) g_{1}\left(x, Q^{2}\right)\right. \\
\left.-\frac{Q^{2}}{\nu} g_{2}\left(x, Q^{2}\right)\right\}, \\
A_{\perp}=\frac{\sigma^{\rightarrow \downarrow}-\sigma^{\leftarrow \downarrow}}{\sigma^{\rightarrow \downarrow}+\sigma^{\leftarrow \downarrow}}=D \cdot E^{\prime} \sin (\theta)\left\{g_{1}\left(x, Q^{2}\right)\right. \\
\left.+\frac{2 E}{\nu} g_{2}\left(x, Q^{2}\right)\right\} .
\end{gathered}
$$

Both, $A_{\|}$and $A_{\perp}$, can be measured by using a longitudinally polarized beam in combination with longitudinally 
and transversely polarized targets. The spin-averaged denominator is expressed in terms of the factor $D$ :

$$
D=\frac{1}{F_{1}\left(x, Q^{2}\right)} \cdot \frac{1}{\nu} \cdot \frac{1-\epsilon}{1+\epsilon R\left(x, Q^{2}\right)} .
$$

Here, $F_{1}\left(x, Q^{2}\right)$ is the unpolarized Dirac form factor and $R\left(x, Q^{2}\right)$ is the ratio $\sigma_{L} / \sigma_{T} . \epsilon$ is a kinematical factor. If $F_{1}$ and $R$ are known, $g_{1}\left(x, Q^{2}\right)$ and $g_{2}\left(x, Q^{2}\right)$ can be simply extracted from eqs. (1) and (2). Although this procedure is quite common in deep inelastic scattering (DIS), it should be pointed out that the unpolarized structure functions are not well known in the resonance region. In the DIS region, i.e. at large values of the invariant mass $W$, the structure functions have been measured quite precisely at CERN and SLAC over a wide range of $x$ and $Q^{2}$.

The GDH integral has gained considerable theoretical and experimental interest in recent years. The sum rule was originally derived for real photons using a few fundamental properties of physics, e.g. causality, unitarity, a low-energy theorem, and an (unsubtracted) dispersion relation. It was shown that the spin-dependent crosssections for real photons scattered off a target with nonzero nuclear spin obey the following relationship:

$$
I\left(Q^{2}=0\right)=\int_{\nu_{\text {thresh }}}^{\infty} \frac{\mathrm{d} \nu}{\nu}\left(\sigma_{\uparrow \downarrow}(\nu)-\sigma_{\uparrow \uparrow}(\nu)\right)=-\frac{2 \pi^{2} \alpha}{M_{N}} \kappa_{N}^{2} .
$$

$\kappa_{N}$ is the anomalous magnetic moment of the target nucleus. The integral extends from the inelastic threshold of the target, i.e. single-pion production threshold in the case of the nucleon, to infinite photon energy. This implies that high-energy contributions to the integral have to be estimated theoretically. It should be noted that the cross-sections are weighted with $1 / \nu$ and therefore low-energy excitations will dominate. Within the last few years, collaborations at MAMI (Mainz) and ELSA (Bonn) measured this integral for the proton using real photons with energies up to $\approx 3.1 \mathrm{GeV}$. First results from MAMI $\left(200 \mathrm{MeV}<E_{\gamma}<800 \mathrm{MeV}\right)$ show that the integral agrees within experimental errors with the expectation of $205 \mu \mathrm{b}$. Therefore, the total contribution from higher photon energies has to be small. An extension of the integral to non-zero values of $Q^{2}$ can be performed by taking the transverse part of the spin-dependent virtual photon cross-sections. Now we have a $Q^{2}$-dependent integral which can be evaluated theoretically using $\chi \mathrm{PT}$ techniques at low $Q^{2}$ and the operator product expansion (OPE) at large $Q^{2}$. Only a small region between about $0.2 \mathrm{GeV}^{2} / c^{2} \lesssim Q^{2} \lesssim 0.5 \mathrm{GeV}^{2} / c^{2}$ cannot be covered using these techniques. Often the difference in the spindependent cross-section is called $\sigma_{T T}^{\prime}$ and is related to the spin structure functions via

$\sigma_{T T}^{\prime}=\frac{1}{2}\left(\sigma_{\uparrow \downarrow}-\sigma_{\uparrow \uparrow}\right)=\frac{4 \pi^{2} \alpha}{M_{N} K}\left(g_{1}\left(x, Q^{2}\right)-\frac{Q^{2}}{\nu^{2}} g_{2}\left(x, Q^{2}\right)\right)$.

We measured the integral for six different values of $Q^{2}$ in a range from $0.02 \mathrm{GeV}^{2} / c^{2}$ to $0.9 \mathrm{GeV}^{2} / c^{2}$ using a polarized ${ }^{3} \mathrm{He}$ target in Hall A at Jefferson Lab. The ${ }^{3} \mathrm{He}$

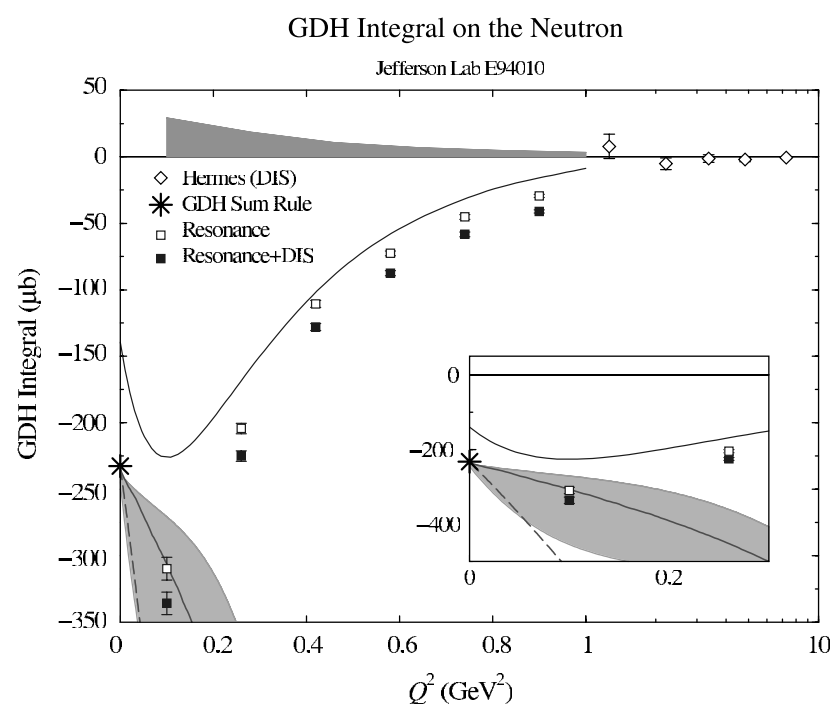

Fig. 1. GDH integral for the neutron. Solid curve: "infrared regulated" $\chi \mathrm{PT}[6]$. The shaded area is an estimate of the theoretical error. Dashed curve: standard $\mathrm{HB} \chi \mathrm{PT}[7]$.

target was operated using the technique of spin exchange with highly polarized Rb. A $40 \mathrm{~cm}$ long thin-walled cylindrical glass container served as the target cell. The target pressure was (10-12) atm and an average in-beam polarization of $0.35 \pm 0.014$ was achieved. Both high-resolution spectrometers (HRS) were used in single-arm mode. The polarization of a longitudinally polarized electron beam $\left(P_{b} \approx 0.70, I=(10-15) \mu A\right)$ was flipped at a rate of $1 \mathrm{~Hz}$. The beam energy varied between $0.86 \mathrm{GeV}$ and $5.06 \mathrm{GeV}$. Data were taken from quasi-elastic kinematics through the resonance region up to an invariant mass $W$ of about $2 \mathrm{GeV}$. We used eq. (5) for the evaluation of the integrals, i.e. we determined the cross-sections and spin structure functions directly from asymmetry measurements. This procedure was necessary since the unpolarized structure functions of the neutron are not well known in the resonance region. We applied the model of degli Atti et al. [4] to extract the neutron information from the ${ }^{3} \mathrm{He}$ data. Radiative corrections were performed using the program POLRAD [5]. Figure 1 shows our results of the GDH integral for the neutron as a function of $Q^{2}$. This is the first measurement of the generalized GDH sum on the neutron in the resonance region. A striking $Q^{2}$-dependence can be observed in this region where the transition from the partonic to the hadronic picture takes place. The shaded region at very low $Q^{2}$ is the prediction of Bernard et al. [6]. The calculation was performed within the framework of "infrared regulated" $\chi \mathrm{PT}$. The shaded region covers the estimated error in the theory due to uncertainties in low-energy constants. The dashed curve is a $\mathrm{HB} \chi \mathrm{PT}$ calculation by Ji and Osborne [7]. It is interesting to note that there has to be a minimum in the GDH integral somewhere between $Q^{2}=0.1 \mathrm{GeV}^{2} / c^{2}$ and 0 . This region will be the focus of a follow-up experiment which will run in 2003 in Hall A at Jefferson Lab [8]. 


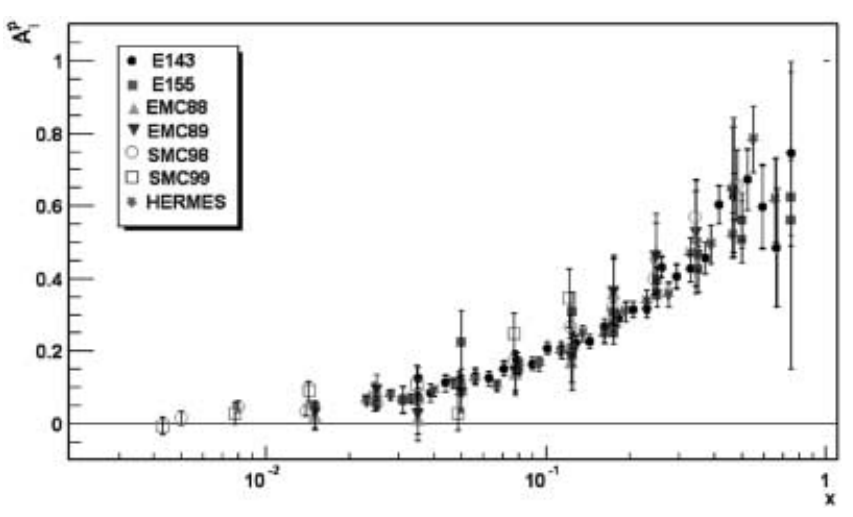

Fig. 2. World data of the virtual photon asymmetry $A_{1}^{p}$ of the proton.

\section{The virtual photon asymmetry $A_{1}^{n}\left(x, Q^{2}\right)$}

Similar to eq. (1), the virtual photon asymmetry, $A_{1}\left(x, Q^{2}\right)$, can be expressed in terms of the spin structure functions via

$A_{1}=\frac{\sigma_{\frac{1}{2}}^{T}-\sigma_{\frac{3}{2}}^{T}}{\sigma_{\frac{1}{2}}^{T}+\sigma_{\frac{3}{2}}^{T}}=\frac{1}{F_{1}\left(x, Q^{2}\right)}\left(g_{1}\left(x, Q^{2}\right)-\frac{4 m_{N}^{2} x^{2}}{Q^{2}} g_{2}\left(x, Q^{2}\right)\right)$.

The 2 nd term in eq. (6) is often kinematically suppressed $\left(\propto 1 / Q^{2}\right)$ and therefore

$$
\begin{aligned}
A_{1}=\frac{g_{1}\left(x, Q^{2}\right)}{F_{1}\left(x, Q^{2}\right)} & =\frac{4 \Delta u+\Delta d}{4 u+d} \text { (proton) }, \\
& =\frac{4 \Delta d+\Delta u}{4 d+u} \text { (neutron) } .
\end{aligned}
$$

Here, we substituted the unpolarized $(u, d)$ and polarized $(\Delta u, \Delta d)$ parton distributions into the structure functions and omitted any sea quark contributions. Experimental data at large $x$ suggest that the $u$-quarks dominate the cross-sections over the $d$-quarks. This can be seen from the large- $x$ behavior of $\frac{F_{2}^{n}}{F_{2}^{p}}$. $A_{1}$ has been measured very precisely over a wide kinematical range for the proton. Figure 2 shows the present quality of the proton data It can be seen that $A_{1}^{p}$ is large and positive as $x \rightarrow 1$. If $A_{1}^{p}$ approaches unity as $x \rightarrow 1$, the asymmetry is dominated by $\frac{\Delta u}{u}$ and therefore $\Delta u=u$. The situation changes for the neutron. The present precision of the data does not allow a reliable prediction for $A_{1}^{n}(x)$ at large values of $x$. For $x \gtrsim 0.3, A_{1}^{n}(x)$ is consistent with zero. This is consistent with a simple $S U(6)$ model prediction for the neutron. However, it is clear from $A_{1}^{p}$ that such simple models are inconsistent with the data, since the $S U(6)$ prediction for the proton is $5 / 9$. Isgur showed that the valence quark $S U(6)$ predictions get modified when hyperfine interactions are taken into account [9]. In this new approach $A_{1}^{p}$ and $A_{1}^{n}$ are expected to approach 1 as $x \rightarrow 1$. Perturbative QCD calculations predict large and positive asymmetries for the proton and neutron at large values of $x$ as well [10].

The experimental conditions in Hall A at Jefferson Lab are an ideal environment to perform precision measurements at large values of $x$, i.e. at small cross-sections. In

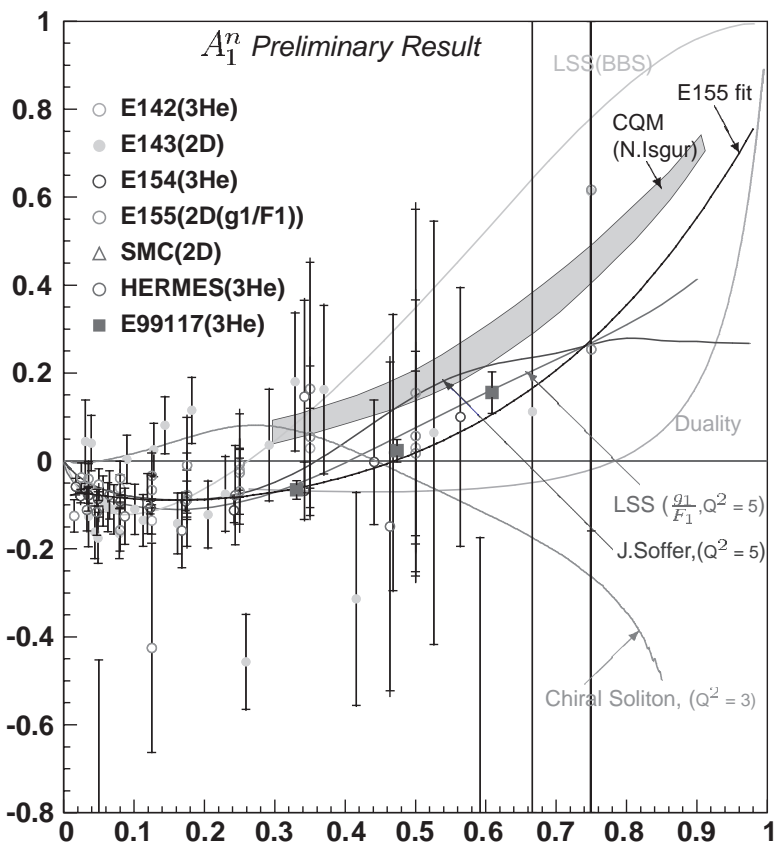

Fig. 3. World data of the virtual photon asymmetry $A_{1}^{n}$ for the neutron as a function of Bjorken $x$. The results from E99-117 are preliminary.

the summer of 2001 the asymmetry $A_{1}^{3} \mathrm{He}$ was measured with unprecedented precision. The target was the same as described in sect. 2. The average target polarization was about 0.40 with beam on target and the beam polarization was about 0.80 . Also here, the method described in ref. [4] was used to extract the neutron asymmetry, $A_{1}^{n}$. The preliminary result is shown in fig. 3. Radiative corrections have been applied using the program POLRAD.

The results of E99-117 show clearly that $A_{1}^{n}$ changes $\operatorname{sign}$ at $x \approx 0.5$ and increases with increasing $x$. The three measured values are below Isgur's extended quark model prediction and indicate a negative contribution of $\Delta d$ to the asymmetry.

\section{Precision measurement of $g_{2}^{n}\left(Q^{2}\right)$ at $\mathrm{x} \approx 0.2$}

As pointed out in the previous sections, the spin structure functions $g_{1}\left(x, Q^{2}\right)$ and $g_{2}\left(x, Q^{2}\right)$ can be extracted from two independent asymmetry measurements. $g_{1}\left(x, Q^{2}\right)$ has a simple interpretation within the quark model picture. It is just the sum of the charge (squared) weigthed polarized parton number densities. $g_{2}\left(x, Q^{2}\right)$, on the other hand, does not have such a simple interpretation. $g_{2}\left(x, Q^{2}\right)$ is related to the transverse spin structure function $g_{T}=g_{1}+g_{2}$ and quark-gluon correlations as well as quark mass terms enter as twist-3 contributions in leading order in $\alpha_{s} . g_{2}$ is called a twist- 3 structure function in the language of the operator product expansion (OPE). Twist-3 contributions to physical observables are suppressed by $1 / Q$. Wandzura and Wilzcek [11] found that the leading-twist, i.e. twist-2, 


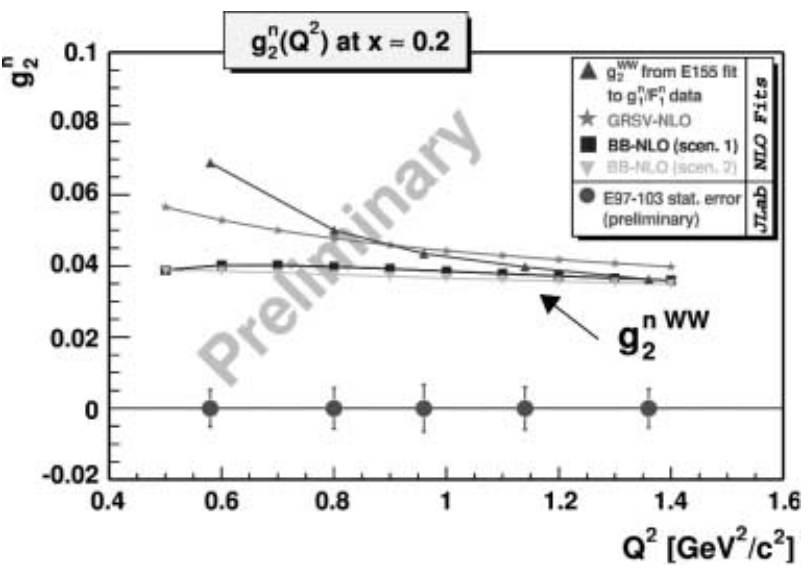

Fig. 4. Statistical precision achieved in E97-103. The solid lines are NLO results for $g_{2}^{\mathrm{WW}}$ for different polarized-quark distributions. The markers indicate the points where the NLO calculations were performed.

part of $g_{2}$ is purely determined by $g_{1}$ via

$$
g_{2}^{\mathrm{WW}}\left(x, Q^{2}\right)=-g_{1}\left(x, Q^{2}\right)+\int_{x^{\prime}}^{1} \frac{\mathrm{d} x^{\prime}}{x^{\prime}} g_{1}\left(x^{\prime}, Q^{2}\right) .
$$

This implies that $g_{2}$ can be decomposed in $g_{2}=g_{2}^{\mathrm{WW}}+$ $g_{2}^{\text {h.t. }}$, where $g_{2}^{\text {h.t. }}$ are all the higher-twist contributions to $g_{2}$. So far the information on $g_{2}^{\text {h.t. }}$ has been very limited. The main experimental direct evidence for non-zero higher-twist contributions comes from the SLAC E155 and E155x experiments [12]. These experiments measured the twist-3 matrix element $d_{2}=3 \int_{0}^{1} \mathrm{~d} x x^{2} g_{2}^{\text {tw } 3}\left(x, Q^{2}\right)$ for the proton and neutron. The results are: $d_{2}^{p}=0.0032 \pm 0.0017$ and $d_{2}^{n}=0.0079 \pm 0.0048$ [12]. The data were taken in a $Q^{2}$ range from $0.7 \mathrm{GeV}^{2} / c^{2}$ to $20 \mathrm{GeV}^{2} / c^{2}$.

Since hardly any additional information is available on $g_{2}^{n}$, the polarized ${ }^{3} \mathrm{He}$ target of Hall A was used to measure the $Q^{2}$-dependence of $g_{2}^{n}$ at nearly constant values of $x(\approx 0.2)$. The range in $Q^{2}$ varied from $1.36 \mathrm{GeV}^{2} / c^{2}$ down to $0.58 \mathrm{GeV}^{2} / c^{2}$. Due to the $1 / Q$ suppression of the twist-3 part, its contribution to $g_{2}$ is expected to increase at lower values of $Q^{2}$. The experiment was performed in 2001. Figure 4 shows the statistical precision we achieved. The errors were reduced by nearly one order of magnitude as compared to previous data at $x=0.2$. The figure also shows some twist-2 expectations for $g_{2}^{\mathrm{WW}}$ in next-toleading order (NLO). Different modern parameterizations for the polarized quark and gluon density distributions served as input. First, DGLAP evolutions to the measured $Q^{2}$ were performed [13] for these distributions, then $g_{1}$ was determined, and finally $g_{2}^{\mathrm{WW}}$ was calculated based on eq. (8). It is interesting to note that all the parameterizations seem to converge to a common value for $g_{2}^{\mathrm{WW}}$ at $Q^{2}$ values $>1 \mathrm{GeV}^{2} / c^{2}$, but some dispersion can be observed at the lower $Q^{2}$ values. This is most likely a consequence of the scale at which the input distributions were parameterized. The new data from Hall A will certainly shed more light on this low- $Q^{2}$ regime.

\section{Conclusions}

The experimental data presented in this paper show the unique opportunity for Jefferson Lab to contribute to our basic understanding of the nucleon spin structure. The combination of a dense, highly polarized ${ }^{3} \mathrm{He}$ target and the intense polarized CEBAF c.w. electron beam allowed us to access and improve the precision of some observables such as the generalized GDH sum rule, $A_{1}^{n}(x)$ at large $x$, and $g_{2}^{n}\left(Q^{2}\right)$ at low $Q^{2}$ and constant $x$. Nuclear corrections are well under control in the DIS region. The corrections in the resonance region were performed using a model of Scopetta et al. [4]. Future experiments on higher-twist observables, spin duality, the electric form factor of the neutron, $G_{E}^{n}$, at large $Q^{2}$, and, especially, Jefferson Lab at $12 \mathrm{GeV}$, promise a lot more precision data on the (spin) structure of the neutron.

This work was supported by the US Department of Energy (DOE) under contracts DE-FG02-99ER41101 and DE-AC0584ER40150.

\section{References}

1. B.W. Filippone, X.D. Ji, Adv. Nucl. Phys. 26, 1 (2001).

2. J.D. Bjorken, Phys. Rev. 148, 1467 (1966); Phys. Rev. D 1, 1376 (1970).

3. S.B. Gersimov, Sov. J. Nucl. Phys. 2, 430 (1966); S.D. Drell, A.C. Hearn, Phys. Rev. Lett. 16, 908 (1966).

4. C.C. degli Atti et al., Phys. Lett. B 44, 223 (1997).

5. I.V. Akushevich et al., J. Phys. G 20, 513 (1994).

6. V. Bernard et al., hep-ph/0203167 (2002).

7. X.D. Ji, J. Osborne, J. Phys. G 27, 127 (2001).

8. JLab prop. E97-110, J.P. Chen, A. Deur, F. Garibaldi.

9. N. Isgur, Phys. Rev. D 59, 034013 (1999).

10. G.R. Farrar, D.R. Jackson, Phys. Rev. Lett. 35, 1416 (1975); S.J. Brodsky et al., Nucl. Phys. B 441, 197 (1995).

11. S. Wandzura, F. Wilczek, Phys. Lett. B 72, 195 (1977).

12. E155 Collaboration (P.L. Anthony et al.), Phys. Lett. B 553, 18 (2003), hep-ex/0204028.

13. M. Hirai et al., Comput. Phys. Commun. 108, 38 (1998). 